\title{
Modifictcación técnica y experiencia con injerto óseo esponjoso de olécranon para el tratamiento die la hendidura alveolar
}

\section{Technical modification and experience with cancellous hone graft from olécranon for alveolar cleft}

Colombia QUINTERO GONZÁLEZ*

Edison QUINTERO GONZÁLEZ*, Juan Carlos PÉREZ QUINTERO**

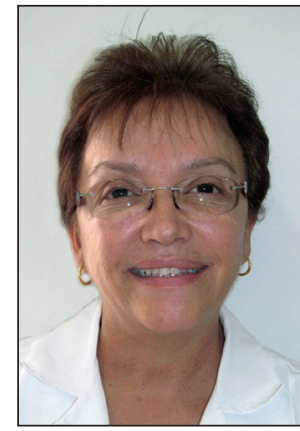

Quintero Gonzáles C.

\section{Resumen}

Antecedentes y Objetivo. Para muchos cirujanos plásticos, el procedimiento quirúrgico de elección para el tratamiento de la hendidura alveolar consiste en tomar como zona donante de hueso la cresta iliaca por ser una técnica fácil y reproducible y por la alta disponibilidad de tejido que proporciona. Es escasa la literatura en cuanto al uso de olecranón como zona donante para el tratamiento de esta patología.

El objetivo de este trabajo es describir los resultados del uso de injerto óseo esponjoso de olécranon para pacientes con hendidura nasoalveolar, recopilando una experiencia de más de 10 años con el procedimiento y valorando la escasa morbilidad generada.

Material y método. Realizamos un estudio descriptivo y transversal sobre una serie de pacientes con diagnóstico de labio y paladar hendido uni o bilateral en los que se utilizó la técnica quirúrgica de toma de injerto esponjoso de olécranon preservando una ventana ósea de cortical para cierre del defecto. Recogimos variables sociodemográficas: edad, sexo, procedencia, diagnóstico, número de injertos; variables clínicas: tiempo de cirugía, dolor postquirúrgico, tiempo de hospitalización y de regreso a actividades cotidianas y complicaciones. Evaluamos a los pacientes clínicamente en el primer control 4 días después de la intervención.

Resultados. Obtuvimos datos de 111 pacientes en los que se realizaron 146 injertos óseos de olécranon. El tiempo quirúrgico de la cirugía osciló desde 1 a 1.5 horas. El regreso a las actividades cotidianas tuvo un rango de 2 a 4 días del postoperatorio. El dolor postoperatorio en la zona donante estuvo presente en 5 pacientes (grado III-IV en escala de dolor según las facies) y solo en el primer y segundo tiempo de injerto. Cuatro de ellos tenían diagnóstico según Veau grado III y 1 grado IV. De esos 5 pacientes con dolor, 4 regresaron a sus actividades cotidianas a los 4 días de postoperatorio y 1 a los 3 días. En el momento del injerto óseo, 1 tenía 4 años de edad, 2 tenían 5 años, 1 tenia 6 años, y otro 8 años.

Conclusiones. Con la modificación propuesta obtenemos únicamente injerto óseo esponjoso preservando la capa cortical para la posterior cobertura de la ventana ósea practicada en el olécranon. Nuestra casuística pone en evidencia el reducido tiempo quirúrgico de la técnica, la presencia de dolor postoperatorio en un porcentaje bajo de pacientes y su regreso a actividades cotidianas en 2-4 días.

Palabras clave Hendidura alveolar, Injerto óseo, Injerto olécranon, Labio hendido, Paladar hendido

\section{Nivel de evidencia científica Recihido [esta versión] Aceptado}

4c Terapéutico 4 septiembre/2018 21 julio/2019
Background and Objective. For many plastic surgeons, procedure of election in the management of alveolar cleft is to take iliac crest as donor area as it is an easy surgical technique, with high availability of tissue and adequate reproducibility. The available literature regarding the use of olécranon as a donor area for alveolar cleft management is scarce.

Our aim is to describe the results of olécranon cancellous bone graft management for patients with nasoalveolar cleft, compiling a more than 10 -year experience performing this procedure and focusing on the low morbidity generated.

Methods. We conduct a descriptive, cross-sectional study on patients with a diagnosis of unilateral or bilateral cleft lip and palate to whom olécranon bone grafts were performed preserving a bone cortical window to close de defect. Sociodemographic variables were collected such as age, sex, origin, diagnosis, number of grafts; and clinical variables such as surgery time, post-surgical pain, time of hospitalization, days of return to daily activities and complications. Patients were clinically evaluated at the first control 4 days after surgery.

Results. Data were obtained from 111 patients, to whom 146 cancellous bone graft from olécranon were performed. Surgical time ranged from 1 to 1.5 hours. Return to daily activities ranged from 2 to 4 days after surgery. Five patients presented post-surgical pain in the donor area (grade III-IV in the pain scale according to the facies)., and only in the first and second time of grafts. Of those patients, 4 were diagnosed as grade III and 1 as grade IV according to Veau. Four returned to daily activities 4 days after surgery and 1 after 3 days. At the time of bone grafting, 1 patient was 4 years old, 2 were 5 years old, 1 was 6 years old, and another one was 8 years old.

Conclusions. With the proposed modification we obtain only cancellous bone graft preserving cortical surface for ulterior close of the bone window created on the olécranon. Our serie of patients evidence a reduced surgical time, the presence of pain only in a low percentage of patients and their return to daily activities in 2-4 days after surgery.

$\begin{array}{lr}\text { Key words } & \begin{array}{l}\text { Alveolar cleft, Bone graft, Olécranon graft, } \\ \text { Cleft palate, Cleft lip }\end{array} \\ \text { Level of evidence } & 4 c \text { Therapeutic } \\ \text { Received [this version] } & 4 \text { September/2018 } \\ \text { Accepted } & 21 \text { July/2019 }\end{array}$

Conflicto de intereses: Los autores declaran no tener ningún interés financiero relacionado con el contenido de este artículo.

Financiación: No hubo fuentes externas de financiación para este trabajo. 
Introducción

Los injertos óseos se han empleado como tratamiento de la hendidura alveolar en pacientes con paladar hendido aproximadamente desde la segunda década del siglo pasado. Actualmente, disponemos de una gran variedad de zonas donantes como la tibia, costilla, calota craneana y cresta ilíaca.

La reconstrucción de la hendidura alveolar con cualquier tipo de injerto óseo debe tener una serie de objetivos esenciales para la adecuada reconstrucción funcional del maxilar, a saber: estabilizar los segmentos maxilares, proporcionar continuidad al arco maxilar, lograr el cierre de la fisura oronasal, otorgar soporte óseo a las piezas dentarias adyacentes a la fisura, mejorar la articulación de la palabra, dar soporte al ala nasal y al labio, mejorar la simetría facial, así como también permitir la rehabilitación fija otorgando tejido óseo suficiente para colocar implantes osteointegrados. Con este fin, la cresta ilíaca como sitio donante se ha utilizado de forma rutinaria para la reconstrucción ósea de la región maxilofacial. ${ }^{(1)}$

Como cualquier otra zona donante, la cresta ilíaca presenta efectos adversos, entre los que los dos principales son, generalmente, el que la edad en la que se realiza la toma del injerto suele ser la etapa de crecimiento y desarrollo del paciente, a lo que se suma la morbilidad asociada a la lesión músculo-tendinosa provocada durante la disección quirúrgica que genera limitación en la deambulación del paciente, con un amplio margen de necesidad de tiempo hasta la recuperación de la actividad habitual. ${ }^{(2,3)}$

La hendidura alveolar es una malformación congénita que va ligada a la fisura ocasionada por el paladar hendido y supone una malformación que afecta directamente a la calidad de vida del paciente. La frecuencia mundial de aparición de esta afectación descrita en la literatura varía ampliamente desde 1 por cada 1.200 a 1 por cada 700 nacidos vivos. ${ }^{(4)}$ En América Latina se reportan 0.87 por cada 1000 nacimientos con labio hendido con o sin paladar hendido, y 0.13 por cada 1000 nacimientos con paladar hendido. ${ }^{(4,5)}$ En Colombia, está comunicada una incidencia de 8.000 nuevos casos por año de malformaciones craneofaciales, de las cuales se identifica una prevalencia de labio hendido con o sin paladar hendido de 8.28 por cada 1000 nacidos, y de paladar hendido de 3.13 por cada $1000 .^{(6)}$

El tratamiento de la fisura alveolar como tal ha tenido cambios a través de los años, con diversos métodos que tratan de proporcionar al paciente los objetivos reconstructivos que se deben lograr en este procedimiento y que hemos mencionado con anterioridad. En el contexto adecuado, el hueso esponjoso autólogo fresco es ideal para el injerto secundario ya que posee una serie de características que lo hacen apto para la reconstrucción de esta zona anatómica, entre las cuales la de mayor relevancia es el aporte de células óseas aptas para la odontogénesis, lo que en la actualidad le convierte en patrón de oro. ${ }^{(7,8)}$

Dentro de nuestra experiencia previa, los pacientes a los que se les realizaba injerto óseo para hendidura alveolar con zona donante de cresta iliaca presentaban en sus controles postquirúrgicos dolor, dificultad en la deambulación, retardo en el regreso a sus actividades cotidianas, larga estancia hospitalaria, requerimiento de analgésicos durante su hospitalización y en su tratamiento ambulatorio.

Existe poca literatura sobre el uso de hueso esponjoso de la región olecraniana como zona donante para injertos alveolares, ${ }^{(9,10)}$ Es por ello que, dado que la búsqueda de la zona donante ideal es un ejercicio académico incesante, nos surgió la inquietud de comprobar la poca morbilidad provocada en los pacientes con el uso de esta técnica y decidimos llevar a cabo el siguiente estudio.

\section{Material y método}

Presentamos un estudio descriptivo y transversal de los resultados del uso de injerto óseo esponjoso de olécranon en pacientes con hendidura alveolar tratados entre 2005 a 2017 en el Hospital Infantil Universitario Rafael Henao Toro de la Ciudad de Manizales, Colombia, por el grupo multidisciplinario de la Clínica de Labio y Paladar Hendido de la institución.

En cada paciente tomamos las variables sociodemográficas como edad, sexo, procedencia, diagnóstico, numero de injertos, y las variables clínicas como tiempo de cirugía, dolor postquirúrgico, tiempo de hospitalización, días de regreso a las actividades cotidianas y complicaciones sufridas. Evaluamos a todos los pacientes clínicamente en el primer control a los 4 días de la intervención $\mathrm{y}$ con todos los hallazgos encontrados, completamos una base de datos de la que extrajimos las conclusiones del presente estudio.

Para la valoración del dolor postoperatorio en la zona donante del injerto óseo empleamos la tabla de caras por ser este un sistema objetivo en Pediatría mediante el cual la madre refiere el comportamiento del paciente tras la cirugía y según este, se determina el grado de dolor del niño.

\section{Técnica quirúrgica}

Elegimos como brazo donante el no dominante del paciente.

Como margen de seguridad dejamos $1 \mathrm{~cm}$ hacia distal del proceso del olécranon. Realizamos una incisión en 
$\mathrm{S}$ itálica o línea recta de aproximadamente $2-4 \mathrm{~cm}$ para la exposición ósea. Diseñamos un bloque óseo tridimensional con forma de trapecio. Llevamos a cabo incisión en el periostio y lo dejamos pediculado a fin de tener una ventana de cobertura vascular para el cierre; a continuación utilizamos un osteotomo delgado para levantar el segmento cortical, y exponemos la porción esponjosa para la toma del injerto que hacemos con cureta delgada. Rellenamos la hendidura alveolar con el injerto y cubrimos el injerto nasoalveolar con colgajos mucoperiósticos gingivales. En la zona donante tomamos la porción cortical del bloque óseo y la colocamos en el defecto óseo de la zona donante, fijándola con sutura reabsorbible y cubriéndola con el colgajo de periostio previamente diseñado. Inmovilizamos el brazo del paciente con férula de yeso hasta control postoperatorio a los 4 días; administramos antibiótico profiláctico durante 3 días.(Fig. 1-5).

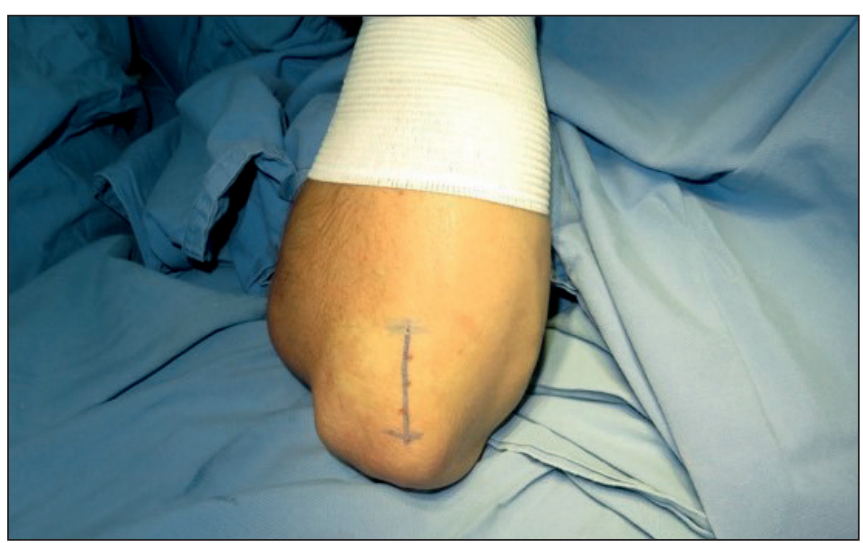

Figura 1. Marcaje en región olecraniana para la toma del injerto óseo.

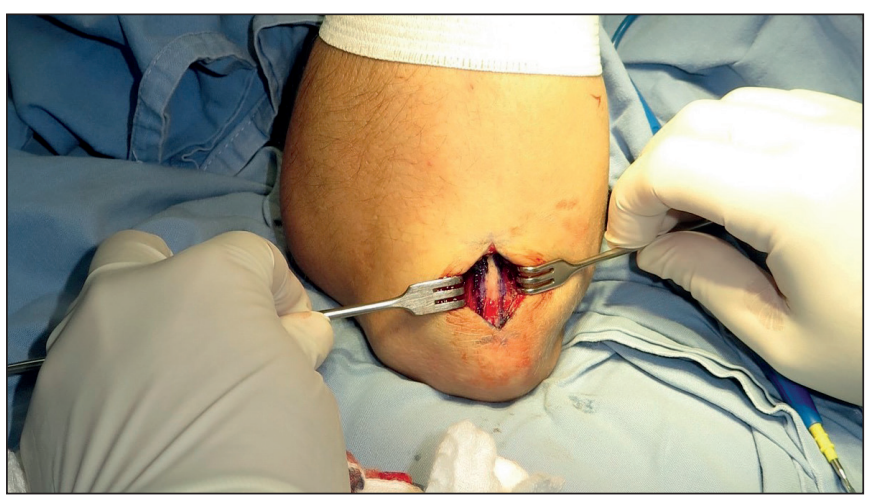

Figura 2. Diseño del bloque óseo para incisión en la cortical.

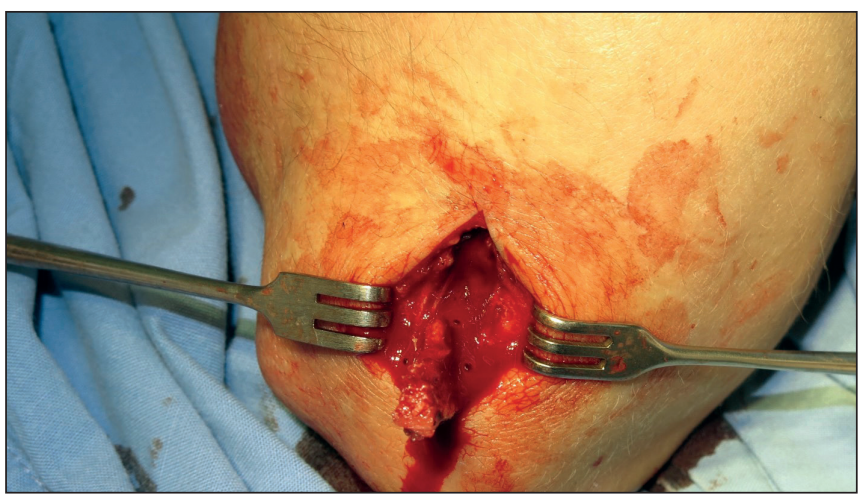

Figura 3. Elevación de ventana de cortical para la toma del injerto esponjoso.

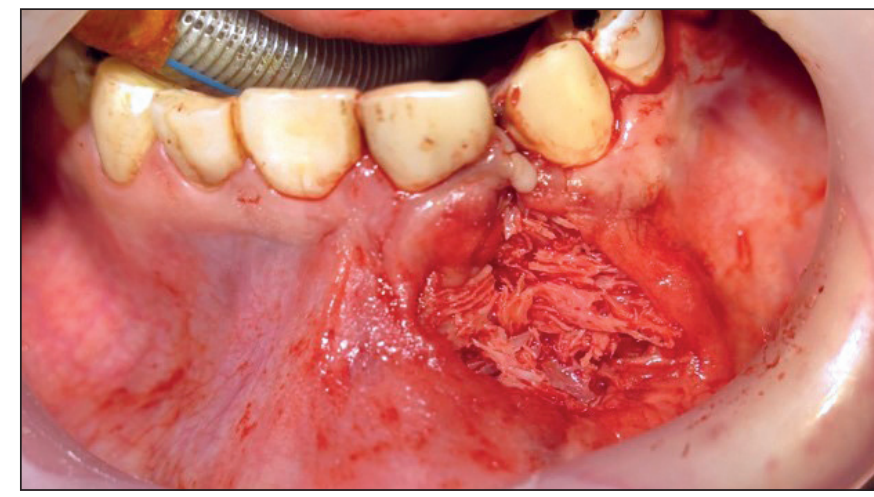

Figura 4. Colocación del injerto de esponjosa en la hendidura alveolar.

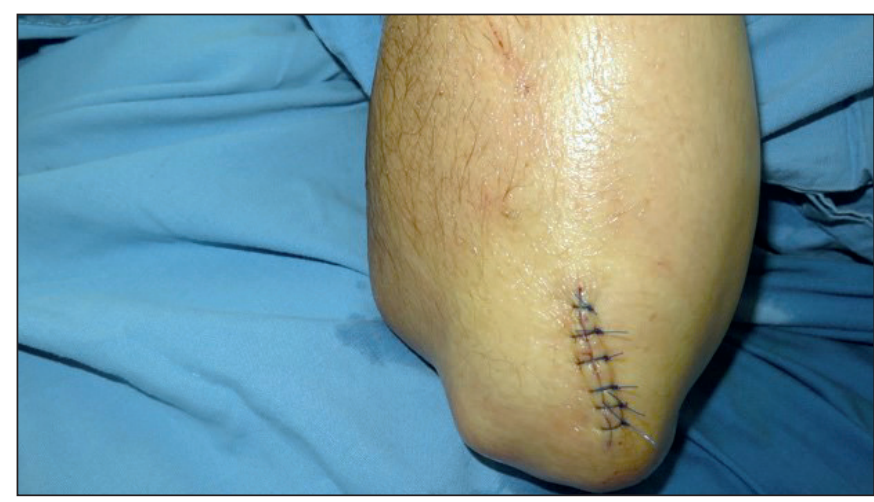

Figura 5. Cierre de la zona donante.

\section{Resultados}

Recopilamos un total de 111 pacientes a los que se les había realizado injerto nasoalveolar con hueso esponjoso del olécranon, 64 (58\%) fueron hombres y 47 (42\%) mujeres (Gráfico 1) con hendidura alveolar grado III de Veau en 83 casos (75\%) y grado IV en $28(25 \%)$ (Tabla I). La edad máxima de los pacientes en el momento de la cirugía fue de 22 años y la mínima de 4 años (media de 10.3 años).

Gráfico 1. Distribución según sexo de los pacientes de nuestro grupo de estudio

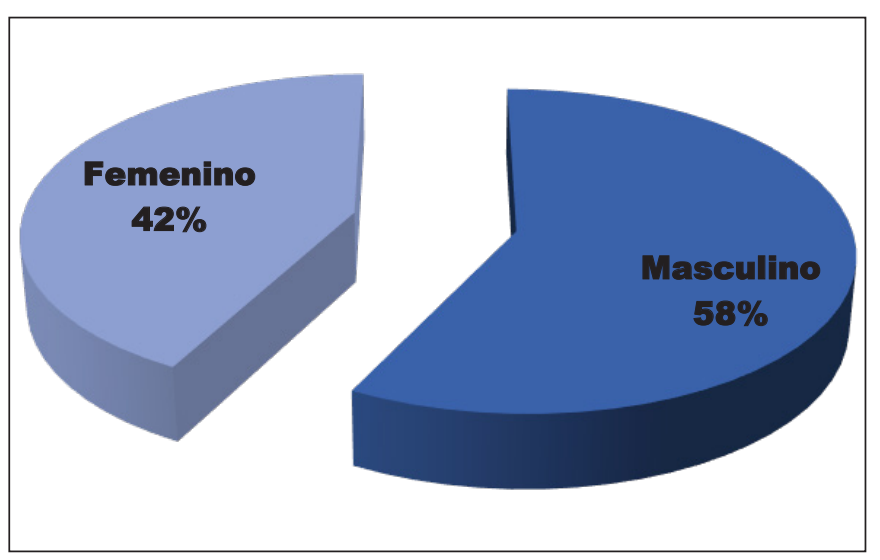

Para distribuir los pacientes según el número de injertos óseos alveolares recibidos, tomamos en cuenta los tiempos quirúrgicos de cada injerto. Así, a 81 pacientes (73\%) se les realizó solo 1 injerto óseo, a 27 pacientes $(24,3 \%) 2$ injertos en diferentes tiempos quirúrgicos, a 2 
Tabla I. Distribución de pacientes según Grado de Veau de la hendidura alveolar

\begin{tabular}{|c|c|c|c|}
\hline GRADO & Frec. & \% & Acum. \\
\hline Grado III & 83 & $75 \%$ & $75 \%$ \\
\hline Grado IV & 28 & $25 \%$ & $100 \%$ \\
\hline TOTAL & $\mathbf{1 1 1}$ & $\mathbf{1 0 0} \%$ & \\
\hline
\end{tabular}

pacientes (1.8\%) 3 injertos óseos, y a 1 paciente $(0,9 \%)$ 5 injertos. Cabe aclarar que todos los pacientes a los que se les realizó 1 solo injerto óseo tenía diagnóstico de paladar hendido unilateral; los pacientes a los que se les efectuaron 2 injertos tenían diagnóstico de paladar hendido bilateral; y los pacientes a los que se les realizaron más de 2 tiempos quirúrgicos presentaron mala higiene oral, poca adherencia al tratamiento ambulatorio y poca red social para ayudarles en sus cuidados (Tabla II).

Tabla II. Distribución de pacientes según número de injertos óseos alveolares realizados para la corrección de su hendidura alveolar

\begin{tabular}{|c|c|c|c|}
\hline $\begin{array}{c}\mathbf{N}^{\mathbf{0}} \mathbf{D E} \\
\text { INJERTOS }\end{array}$ & Frec. & $\%$ & Acum. \\
\hline 1 injerto & 81 & $73 \%$ & $73 \%$ \\
\hline 2 injertos & 27 & $24.3 \%$ & $97.3 \%$ \\
\hline 3 injertos & 2 & $1.8 \%$ & $99.1 \%$ \\
\hline 5 injertos & 1 & $0.9 \%$ & $100 \%$ \\
\hline TOTAL & $\mathbf{1 1 1}$ & $\mathbf{1 0 0} \%$ & \\
\hline
\end{tabular}

En cuanto a la estancia hospitalaria para el primer injerto, 77 pacientes (69\%) fueron ambulatorios y dados de alta hospitalaria tras la recuperación de la anestesia; $34(31 \%)$ permanecieron ingresados durante 1 día por pertenecer a otros municipios o a otros departamentos (Tabla III).

Tabla III. Estadística de estancia hospitalaria en el primer injerto óseo alveolar

\begin{tabular}{|c|c|c|c|}
\hline $\begin{array}{c}\text { DÍAS DE } \\
\text { ESTANCIA }\end{array}$ & Frec. & $\%$ & Acum. \\
\hline Ambulatorios & 77 & $69 \%$ & $69 \%$ \\
\hline 1 día & 34 & $31 \%$ & $100 \%$ \\
\hline TOTAL & $\mathbf{1 1 1}$ & $\mathbf{1 0 0} \%$ & \\
\hline
\end{tabular}

En cuanto al tiempo quirúrgico para el primer injerto, el $90 \%$ de los casos fueron realizados entre 1.05 a 1.15 horas: 1 hora en 6 pacientes (5.4\%), 1.05 horas en 45 pacientes (40.5\%), 1.10 horas en 33 pacientes $(29.7 \%)$, 1.15 horas en 24 pacientes $(21.7 \%)$ y 1.30 horas en 3 pacientes $(2.7 \%)$ (Tabla IV)

\begin{tabular}{|} 
Tabla IV. Distribución del tiempo quirúrgico \\
para el primer injerto \\
\begin{tabular}{|c|c|c|c|}
\hline $\begin{array}{c}\text { TIEMPOS } \\
\text { QUIRÚRGICOS } \\
\mathbf{1}^{\text {er }} \text { INJERTO }\end{array}$ & Frec. & $\%$ & Acum. \\
\hline 1 hora & 6 & $5.4 \%$ & $5.4 \%$ \\
\hline 1.05 horas & 45 & $40.5 \%$ & $45.9 \%$ \\
\hline 1.10 horas & 33 & $29.7 \%$ & $75.7 \%$ \\
\hline 1.15 horas & 24 & $21.7 \%$ & $97.3 \%$ \\
\hline 1.30 horas & 3 & $2.7 \%$ & $100 \%$ \\
\hline TOTAL & $\mathbf{1 1 1}$ & $\mathbf{1 0 0} \%$ & \\
\hline
\end{tabular}
\end{tabular}

Evaluamos la presencia de dolor postoperatorio en el área donante desde el postquirúrgico inmediato hasta la primera consulta a los 4 días del procedimiento. Manifestaron dolor 5 pacientes $(4.5 \%)$ y 106 no (95.5\%) (Tabla V).

Tabla V. Evaluación del dolor postquirúrgico del área donante hasta la primera consulta 4 días después del procedimiento

\begin{tabular}{|c|c|c|c|}
\hline $\begin{array}{c}\text { PRESENCIA } \\
\text { DE DOLOR EN } \\
\text { OLÉCRANON }\end{array}$ & Frec. & $\%$ & Acum. \\
\hline NO & 106 & $95.5 \%$ & $95.5 \%$ \\
\hline SI & 5 & $4.5 \%$ & $100 \%$ \\
\hline TOTAL & $\mathbf{1 1 1}$ & $\mathbf{1 0 0} \%$ & \\
\hline
\end{tabular}

En cuanto al total de complicaciones, recogimos 12: dolor en los 5 casos referidos, emesis en 5 casos y tos en 2 pacientes.

Evaluamos la incapacidad y morbilidad que genera la zona donante mediante el dolor y el regreso a actividades cotidianas, comprobando que la mayoría necesitó entre 2 a 4 días y detallando los siguientes resultados: regreso a las actividades cotidianas académicas y laborales a los 2 días en 29 pacientes (26.2\%), a los 3 días en 37 pacientes $(33.3 \%)$ y a los 4 días en 45 pacientes (40.5\%). (Tabla VI) 
Tabla VI. Valoración de los días hasta regreso de los pacientes a sus actividades cotidianas tras el procedimiento quirúrgico

\begin{tabular}{|l|c|c|c|}
\hline $\begin{array}{c}\text { REGRESO } \\
\text { A VIDA } \\
\text { COTIDIANA }\end{array}$ & SI & NO & TOTAL \\
\hline 2 días & 29 & $26.2 \%$ & $26.2 \%$ \\
\hline 3 días & 37 & $33.3 \%$ & $59.5 \%$ \\
\hline 4 días & 45 & $40.5 \%$ & $100 \%$ \\
\hline TOTAL & $\mathbf{1 1 1}$ & $\mathbf{1 0 0} \%$ & \\
\hline
\end{tabular}

Tabla VII. Cruce de datos entre la variable dolor y el número de injertos realizados en el paciente

\begin{tabular}{|l|c|c|c|}
\hline \multirow{2}{*}{ N$^{0}$ DE INJERTOS } & \multicolumn{2}{|c|}{ DOLOR } & \multirow{2}{*}{ TOTAL } \\
\cline { 2 - 3 } & NO & SI & \\
\hline Primer injerto & 79 & 2 & $\mathbf{8 1}$ \\
\hline Segundo injerto & 24 & 3 & $\mathbf{2 7}$ \\
\hline Tercer injerto & 2 & 0 & $\mathbf{2}$ \\
\hline Quinto injerto & 1 & 0 & $\mathbf{1}$ \\
\hline TOTAL & $\mathbf{1 0 6}$ & $\mathbf{5}$ & $\mathbf{1 1 1}$ \\
\hline
\end{tabular}

Con las variables obtenidas decidimos realizar varios cruces para evaluar los comportamientos del dolor por ser esta la morbilidad más significativa en la evaluación de la zona donante.

Al cruzar la variable dolor con el número de injertos realizados en cualquier tiempo quirúrgico encontramos que 2 pacientes presentaron dolor en el primer injerto y 3 pacientes en el segundo injerto (Tabla VII). De los 5 pacientes que presentaron dolor, 4 de ellos tenían

Gráfico 2. Cruce de datos entre la variable dolor y grado Veau III y IV del defecto alveolar de los pacientes

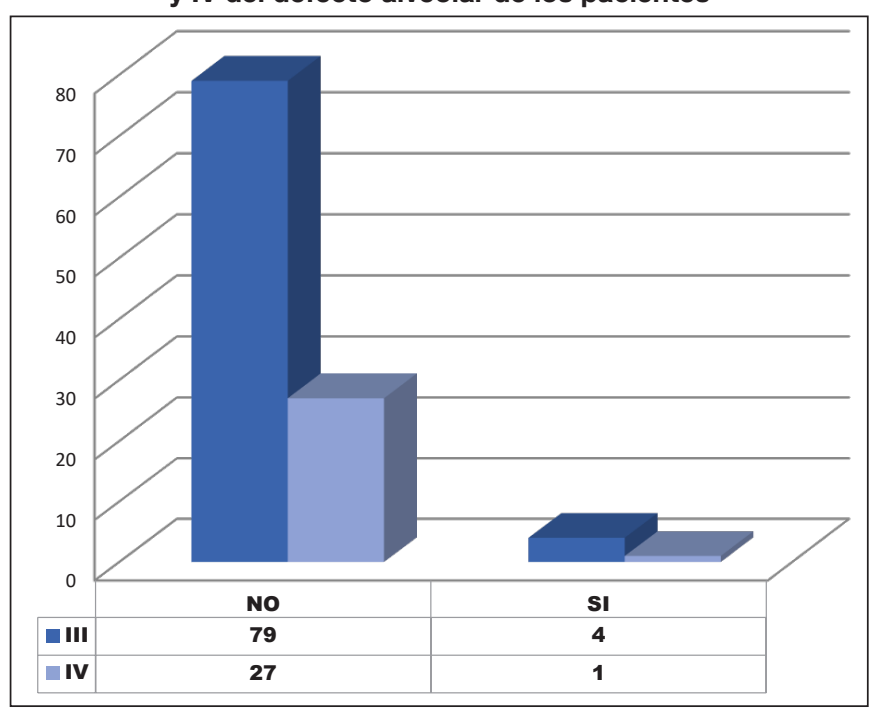

Gráfico 3. Cruce de datos entre la variable dolor y los días hasta el regreso a las actividades cotidianas de los pacientes tras la intervención quirúrgica

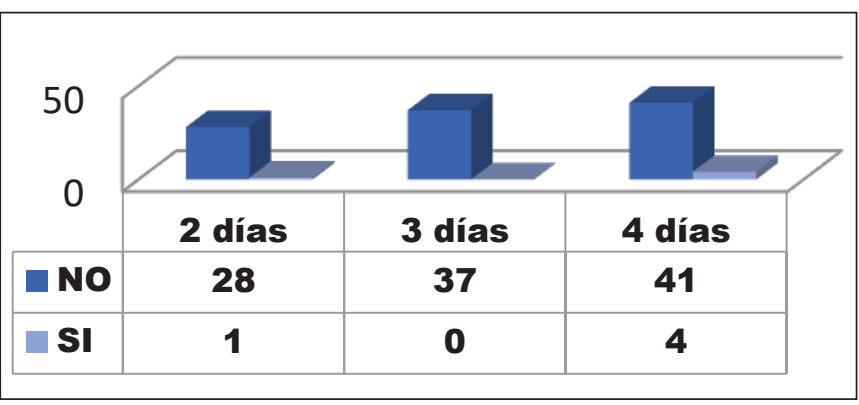

diagnostico según Veau grado III y 1 paciente grado IV (Gráfico 2). De los 5 pacientes que presentaron dolor, 4 de ellos regresaron a sus actividades cotidianas a los 4 días del tratamiento quirúrgico y 1 a los 3 días (Gráfico 3). En el momento del injerto óseo, 1 paciente de este grupo que presentó dolor tenía 4 años de edad, 2 tenían 5 años, 1 tenía 6 años y otro 8 años.

Discusión

Destacamos en nuestro estudio que, con la modificación técnica que realizamos, únicamente obtenemos el injerto óseo esponjoso preservando la capa cortical ósea para la posterior cobertura de la ventana ósea practicada en el olecranón, lo que a nuestro juicio y según los datos recogidos, mejora la recuperación postoperatoria de los pacientes y reduce la morbilidad de la cirugía, principalmente en cuanto a dolor postoperatorio. Solo un $4.5 \%$ de nuestros pacientes presentaron dolor postoperatorio en la zona donante en grado bajo, que no impidió la adecuada integración a sus actividades cotidianas como regreso al jardín, escuela o colegio sin dolor en un plazo máximo de 4 días en 4 de los 5 pacientes que presentaron dolor y en 1 día en 1 paciente; el resto de pacientes injertados no presentaron dolor en ningún tiempo quirúrgico.

Si bien el concepto de injerto óseo para tratamiento de la hendidura alveolar se introdujo en el año 1900, no fue ampliamente reconocido hasta medio siglo más tarde. A partir de 1955, los reportes literarios de varios centros de Cirugía Plástica europeos evidenciaron el éxito del injerto corticoesponjoso para hendidura alveolar en dos diferentes etapas, la infancia y la niñez. ${ }^{(11,12)}$

Existen múltiples zonas donantes ampliamente referidas en la literatura. La condensación de todas éstas nos sugiere que en la actual práctica quirúrgica de Cirugía Plástica Pediátrica y Craneofacial la elección preferente del sitio donante del injerto óseo esponjoso es el hueso ilíaco, del cual se pueden obtener grandes cantidades de injerto con una técnica quirúrgica efectiva y fácilmente reproducible. ${ }^{(12)}$ Otras regiones donantes utilizadas incluyen la calota, la sínfisis mandibular y la costilla, 
entre otras. Sin embargo, hay que mencionar que su uso generalmente es como segunda o tercera opción más que como indicación inicial por diferentes razones. En primera instancia la tabla externa del cráneo, por su mayor tiempo quirúrgico y mayor potencial de complicaciones. En segunda instancia la sínfisis mandibular por riesgo de lesión del nervio mentoniano y el mayor tiempo, así como por la limitada cantidad de hueso disponible. Finalmente, y no menos importante, el injerto óseo adquirido de hueso y cartílago costales, por riesgo de perforación pleural y cicatriz visible.

Cada zona donante, incluyendo el hueso de cresta ilíaca, tiene complicaciones potenciales, por lo tanto, el sitio óptimo donante sigue siendo objeto de debate. ${ }^{(13,14)}$ En nuestra experiencia optamos por tomar la decisión de buscar zonas donantes alternativas que pudieran aportar hueso esponjoso con la menor morbilidad posible para el paciente, lo que motivó considerar la zona olecraniana como fuente fiable, segura, técnicamente reproducible, de fácil aprendizaje y sobre todo, que no generara repercusiones funcionales o eventualidades más allá de las descritas para los otros sitios donantes.

El manejo quirúrgico de la hendidura alveolar consiste principalmente en injertar en ella hueso esponjoso, proporcionándole luego una adecuada cobertura con colgajos locales para garantizar la vitalidad del injerto. El tratamiento quirúrgico en sí mismo se divide según la edad del paciente en dos fases: reparación primaria y secundaria. La primaria comprende la confección del procedimiento terapéutico desde el nacimiento hasta los 2 años de edad del paciente. La secundaria es en la actualidad la de mayor uso ya que aporta los mejores resultados postquirúrgicos. Esta se divide a su vez en: temprana, convencional o tardía, teniendo en cuenta el tiempo de erupción dental del paciente. La temprana se realiza entre la erupción dentaria primaria y antes de la erupción dentaria permanente, por lo que los pacientes tienen entre 2 a 6 años de edad; la convencional se realiza durante la erupción mixta, es decir entre los 6 a los 12 años de edad; y la tardía, también llamada terciaria, es posterior a la erupción de la dentición permanente. ${ }^{(15-18)}$ Nuestro protocolo de tratamiento opta por realizar injertos óseos por primera vez durante la dentición mixta, que por lo general fue entre los 5 y 12 años, corroborada por la media de edad de los pacientes de nuestro estudio que fue 10.3 años; también realizamos injertos en edades más tempranas, aunque esta no fue la constante en nuestro grupo de estudio, y en edades mas tardías debido a absorción y a otros factores que hacían precisa la indicación del injerto.

La literatura mundial al respecto del uso de injertos de olécranon para el tratamiento de la hendidura alveolar, hasta donde nosotros hemos podido comprobar, es escasa. Encontramos 2 trabajos publicados por Nadal y Bruno $^{(19,20)}$ que evalúan el uso de injertos óseos de esponjosa proponiendo una fuente alternativa a la cresta ilíaca para la obtención de material a injertar; sin embargo, hasta el momento y en lo que respecta a nuestra búsqueda utilizando como palabras clave: injerto óseo, hendidura alveolar, labio hendido y paladar hendido en las bases de datos PubMed, ScienceDirect, ClinicalKey, y UptoDate, si bien obtuvimos información, no encontramos estudios que evaluaron la morbilidad postquirúrgica tomando como referencia el dolor postoperatorio en la zona donante del injerto, el regreso del paciente a sus actividades cotidianas y la duración de la estancia hospitalaria, tal y como hicimos nosotros en el presente estudio. Nadal y Dogliotti ${ }^{(19)}$ reportan un $93.9 \%$ de osteointegración y un $91.8 \%$ de erupción espontánea del canino en la hendidura alveolar reconstruida logrando excelentes resultados postquirúrgicos. Motivados por los adecuados resultados obtenidos por Nadal y Dogliotti en cuanto a eficacia reconstructiva teniendo en cuenta osteointegración y erupción dental, en el presente estudio buscamos resultados en cuanto a la eficacia en la morbilidad causada en la zona donante, obteniendo resultados satisfactorios con muy baja tasa de dolor y bajo tiempo de reingreso a las actividades cotidianas sin dolor, lo cual supuso un aliciente para incorporar esta técnica a nuestro habitual manejo reconstructivo de esta patología.

En cuanto a la técnica quirúrgica que empleamos, se basa en los parámetros dados por Dogliotti ${ }^{(19)}$ con la modificación que aportamos y que consiste en obtener únicamente injerto óseo esponjoso a la vez que preservamos la capa cortical ósea para posteriormente dar cobertura a la ventana ósea practicada en la región olecraniana para la obtención del injerto.

En este estudio sobre 111 pacientes determinamos los siguientes factores sociodemográficos; el $64 \%$ fueron varones, mostrando por tanto una mayor incidencia de la afectación en este sexo. Asimismo, hubo mayor cantidad de pacientes provenientes de zona urbana (97\%) que de áreas rurales; en este punto, probablemente haya un sesgo debido a la dificultad administrativa que tiene nuestro sistema de salud para direccionar a los pacientes y la poca asistencia y continuidad del tratamiento que, por diferentes razones, tienen los pacientes de nuestro ámbito geográfico.

En lo que concierne a la estancia hospitalaria, aunque este procedimiento es de carácter ambulatorio, los pacientes de nuestro grupo de estudio a los que se les dio manejo postquirúrgico intrahospitalario fue de máximo 1 día (37\%) y comprobamos que fueron aquellos que procedían de otros municipios, por lo cual quedaron hospitalizados tras la intervención para evitar así viajes lar- 
gos y nocturnos en pacientes de corta edad. De no ser por este dato, la cirugía habría sido llevada a cabo en todos los casos de forma ambulatoria. El tiempo quirúrgico de la cirugía osciló entre 1 hora a 1 hora y media. De la totalidad de pacientes, el $90 \%$ requirieron de 1.05 a 1.15 horas. El regreso a las actividades cotidianas sin dolor tuvo un rango de 2 a 4 días desde la intervención, lo cual denota una zona donante con morbilidad muy baja y alteración funcional mínima. En lo correspondiente al dolor postquirúrgico en la zona donante, en nuestro estudio solo encontramos 5 pacientes que presentaron dolor, de los cuales todos fueron grado III-IV en la escala de dolor empleada. ${ }^{(20,21)}$ Además, en este grupo, la presencia de dolor se dio en el primer y segundo tiempos quirúrgicos para colocación de injertos óseos, sin que hubiera registro de dolor en posteriores procedimientos; a pesar de este factor dolor, 4 de ellos regresaron a sus actividades cotidianas a los 4 días de la intervención y 1 a los 3 días.

Otras complicaciones que encontramos en el postoperatorio además de dolor fueron 5 pacientes con emesis y 2 con tos, que creemos fueron debidas al efecto de los medicamentos anestésicos más que al procedimiento quirúrgico en sí.

Con este estudio logramos determinar las ventajas que tiene la región olecraniana como fuente donante de injertos óseos esponjosos para el tratamiento quirúrgico de la hendidura alveolar, como son; escaso tiempo quirúrgico, poca estancia hospitalaria, pronto regreso a actividades cotidianas, y sobre todo, poca presentación de dolor postquirúrgico de bajo y muy bajo grado, con lo cual creemos que es una zona donante óptima para este procedimiento.

Aportamos además a la literatura un registro de la escasa morbilidad de la técnica con la modificación técnica que proponemos, y por tanto, exponemos el procedimiento para su inclusión dentro del armamentario de los cirujanos plásticos en el tratamiento de la hendidura alveolar.

\section{Conclusiones}

Describimos la región olecraniana como zona donante de hueso esponjoso y explicamos su uso para el tratamiento quirúrgico corrector de la hendidura alveolar. Destacamos la modificación técnica realizada para la obtención únicamente de injerto óseo esponjoso preservando la capa cortical ósea para la posterior cobertura de la ventana ósea creada en el olécranon para la obtención del injerto, que a nuestro juicio, y según los datos recogidos en nuestra serie de pacientes, favorece una baja tasa de dolor postquirúrgico, reduce la necesidad de estancia hospitalaria y reduce la incidencia de complicaciones, permitiendo el regreso rápido y sin alteraciones del paciente a sus actividades cotidianas.

Es por todo ello que recomendamos la inclusión de esta zona donante como recurso para la toma de injertos óseos alveolares.

\section{Dirección del autor}

Dra. Colombia Quintero González

Servicio de Cirugía Plástica

Hospital Infantil Universitario Rafael Henao Toro

Cra. 23 \#49-30

Manizales, Colombia

Correo electrónico: coloquin@hotmail.com

\section{Bibliografía}

1. Cho G, García E, Nunes R, Marti C, Sieira R, Rivera A. Review of secondary alveolar cleft repair. Ann Maxillofac Surg. 2013; 3(1):46-50.

2. Rawashdeh M, Telaf $\mathbf{H}$. Secondary alveolar bone grafting: the dilemma of donor site selection and morbidity. Br J. Oral Maxillofac Surg. 2008; 46(8):665-670.

3. Vura N, Reddy K, Kaluvala V. Donor site evaluation: anterior iliac crest following secondary alveolar bone grafting. J. Clinic Diagn Res. 2013; 7(11):2627-2630.

4. Menegotto BG, Salzano FM. Epidemiology of oral clefts in a large South American sample. Cleft Palate Craniofac J. 1991;28(4): 373-376.

5. López Palacio AM, Uribe Álvarez C, Aristizabal Puerta GM, Cerón Zapata AM. A Retrospective characterization study on patients with oral clefts in Medellín, Colombia, South America. Perspectiva general de la enfermedad/trastorno. Revista Facultad de Odontología, Medellín, Colombia. 2010: 81.

6. Zarante I, López MA, Caro A, García-Reyes JC, Ospina JC. Impact and risk factors of craniofacial malformations in a Colombian population. International J.of Ped. Otorhinolaryngol. 2009; 73:1434-1437.

7. Eichhorn W, Blessmann M, Pohlenz P, Blake F, Gehrke G, Schmelzle R. Primary osteoplasty using calvarian bone in patients whit cleft lip, alveolus and palate. J. Craniomaxillofac Sug. 2009;37(8):429-433.

8. Janssen N, Weijs W, Koole R, RosenbergA, Meijer G. Tissue engineering strategies for alveolar cleft reconstruction: a systematic review of the literature. Clinic Oral Invest. 2014;18(1):219-226. Hermann NV, Kreiborg S, Darvann TA, Jensen BL, Dahl BL, Bolund S. Early craniofacial morphology and growth in children with unoperated isolated cleft palate. Cleft Palate Craniof J. 2002; 39(6):604.

9. Bruno RJ, Cohen MS, Berzins A, et al. Bone graft harvesting from the distal radius, Olécranon, and iliac crest: a quantitative analysis. J Hand Surg. 2001; 26A:135-141.

10. OMS Asociación Internacional para el estudio del dolor, 2005: Definición de dolor. Ginebra, Suiza.

11. Cricchio G, Lundgren S. Institutionen för odontologi, Käkkirurgi, Medicinska fakulteten, Umeå universitet. Donor Site Morbidity in Two Different Approaches to Anterior Iliac Crest Bone Harvesting. Clinical Implant Dentistry and Related Research. 2003; 5:161-169

12. Eppley BL, Sadove AM. Management of alveolar cleft bone grafting--state of the art. The Cleft Palate-Craniofacial Journal. 2000; 37:229-233. 
13. Zouhary KJ Bone Graft Harvesintg from Distant Sites: Concept and Techniques. Oral Maxillofacial Surg Clinic. N Am. 2010:301-316.

14. Seifeldin SA. Is alveolar cleft reconstruction still controversial? Review of literature. The Saudi Dental Journal.2016;28:3-11.

15. Wolfe SA, Price GW, Stuzin JM, Berkowitz S. Alveolar and Anterior Palatal Clefts. In: McCarthy JG. Plastic Surgery. 1 ed. Philadelphia. W.B. Saunders Company; 1990. Pp. 2753-2770.

16. Daw JL, Patel PK. Management of alveolar clefts. Clin Plastic Surg. 2004;31: 303- 313.

17. Bajaj AK, Wongworawat AA, Punjabi A. Management of Alveolar Clefts. J Craniofac Surg. 2003;14:840-846.
18. Rychlik D, Wójcicki P. Bone graft healing in alveolar osteoplasty in patients with unilateral lip, alveolar process, and opalate clefts. J Craniofac Surg. 2012; 23(1):118-123.

19. Nadal E, Sabás M, Dogliotti P, Espósito R. Secondary alveolar bone grafting: our experience with Olécranon bone graft. $J$ of Craniofacial Surgery. 2010; 21:371-374.

20. Miró J, Castarlenas $\mathbf{E}$, Huguet A. Evidence for the use of a numerical rating scale to assess the intensity of pediatric pain. Eur. J of Pain. 2009; 13:1089-1095.

21. Hicks CL, von Baeyer CL, Spafford PA, van Korlaar I, Goodenough B. The Faces Pain Scale-Revised: toward a common metric in pediatric pain measurement. Pain. 2001;93:173-183.

\title{
Comentario al artículo "Modifictcación técnica y experiencia con injerto óseo esponjoso de olécranon para el tratamiento de la hendidura alveolar"
}

\author{
Héctor O. MALAGÓN HIDALGO \\ Cirujano Plástico, Jefe del Servicio de Cirugía Plástica. Centro Médico Issemym, Metepec, México. Subdirector del Capítulo \\ de Cirugía Craneofacial de FILACP 2018-2020.
}

En el presente artículo los autores nos comparten su experiencia con la técnica de injerto óseo esponjoso de olécranon para el manejo de fisuras alveolares como alternativa al uso de hueso esponjoso de cresta iliaca. De manera atinada, los autores mencionan el dolor postoperatorio como el principal efecto secundario de esta técnica, sin embargo, también es frecuente observar otras complicaciones como son sangrado, lesión nerviosa que causa hipoestesias en el muslo e incluso lesiones en órganos intraabdominales.

Con base en los resultados presentados es evidente la reducción en la intensidad y duración del dolor postoperatorio, logrando así el objetivo de la modificación propuesta en el modelo de tratamiento. Llama la atención el número de procedimientos realizados por caso, por lo que sería importante precisar la razón de realizar múltiples injertos en los pacientes descritos ya que el injerto de cresta iliaca regularmente proporciona resultados adecuados en un solo tiempo quirúrgico. Sería interesante conocer si, en opinión de los autores, esto se debe a la limitación del volumen de hueso obtenido del olécranon o bien si lo realizan así de forma intencional por alguna otra situación especial que pudieran puntualizar en sus comentarios, ya que no se menciona el volumen de hueso esponjoso promedio que es posible obtener con la técnica propuesta.

Como alternativa a las técnicas convencionales de injerto óseo se han propuesto opciones como los chips de hueso liofilizado o bien el uso de sustitutos en forma de pasta o geles. ${ }^{(1-3)}$ En los últimos años he usado la pasta de sulfato de calcio para este fin. Este material proporciona una adecuada matriz para el crecimiento de células me- senquimales y osteoprogenitoras que inician la proliferación y diferenciación en osteoblastos, que sustituyen el material del injerto creando hueso nuevo que mantiene el volumen original a lo largo del tiempo. El principal inconveniente de este material es el costo, aunque en mi opinión, a día de hoy, debería ser considerado como una alternativa principal de tratamiento ya que reduce la necesidad de grandes disecciones en el maxilar, reduciendo por tanto la interferencia en el crecimiento ántero-posterior del mismo, y a la vez elimina la necesidad de un sitio donador, permitiendo una recuperación rápida.

En cualquier caso, la técnica presentada me parece una alternativa muy interesante al uso de injerto de cresta iliaca, teniendo en cuenta la reincorporación prácticamente inmediata de los niños a sus actividades y la nula morbilidad que existe al descartar el dolor postoperatorio; es además una técnica aparentemente fácil de reproducir, por lo que no me queda más que felicitar a la Dra. Quintero y col. por el interés y el seguimiento de sus pacientes y por compartirnos su experiencia con una serie tan importante.

Bibliografía

1. Fairbairn P, Leventis M, Mangham C, Horowitz R. Alveolar Ridge Preservation Using a Novel Synthetic Grafting Material: A Case with Two-Year Follow-Up. Case Rep Dent. 2018, 1;2018:6412806. doi: 10.1155/2018/6412806.

2. Bonzani J, George M, Stevens $\mathbf{M}$. Novel materials for bone and cartilage regeneration. Current Opinion in Chemical Biology. 2006, 10 (6): 568-575.

3. Horowitz R, Leventis M, Rohrer M, Prasad H, Bone Grafting: History, Rationale, and Selection of Materials and Techniques. Compendium on continuing education in dentistry. 2014, 35(4):1-13. 


\section{Respuesta al comentario del Dr. Malagón Hidalgo}

\section{Colombia QUINTERO GONZÁLEZ}

Agradecemos al Dr. Malagón por hacer critica a nuestro estudio; desarrollaremos la discusión y esperamos sea de claridad suficiente para responder al comentario establecido.

Respecto al número de injertos que se realizaron en los pacientes, se llevó a cabo 1 tiempo de injertos en 81 pacientes, 27 pacientes recibieron 2 injertos, 2 pacientes recibieron 3 injertos óseos, y 1 paciente recibió 5 injertos.

Es pertinente mencionar que los pacientes a los que se les realizaron 2 injertos fue porque 25 tenían diagnostico de hendidura bilateral y 2 de hendidura unilateral que además habían recibido tratamiento en otra institución con resultados no satisfactorios, por lo que se debían reinjertar. A 3 pacientes se les realizaron más de 2 injertos, lo cual en nuestra propia autocrítica, también nos llamó la atención.

Por su parte, los 2 pacientes que recibieron 3 tiempos de injertos alveolares tenían el diagnostico de hendidura palatina bilateral y venían remitidos de otro centro donde se les habían practicado tratamiento previo, pero en nuestro concepto, era necesario realizar 2 tiempos de injertos alveolares debido al poco marco alveolar del que disponían para continuar con el manejo integral.

En cuanto al paciente que fue injertado en 5 ocasiones, es oportuno señalar que tenía diagnóstico de hendidura bilateral y había recibido tratamiento extrainstitucional previo con injertos alveolares bilaterales, ambos con malos resultados. En nuestro Servicio se le realizó tratamiento de hendidura bilateral, sin embargo, el paciente era poco adherente al manejo postoperatorio, tenía una mala red de apoyo y poco cuidado postquirúrgico del sitio operatorio, lo cual desencadenó que se perdiera uno de los injertos y que fuera necesario planear el reinjerto varios años después, cuando regresó al hospital.

En nuestra opinión, la realización de injertos en más de 2 ocasiones fue debida más a factores externos que a factores intrínsecos quirúrgicos.

Coincidimos con el Dr. Malagón en que con el injerto de olécranon disponemos de menos volumen de injerto esponjoso en comparación con el obtenido de la cresta iliaca; sin embargo, se está adelantando un estudio volumétrico en nuestra institución para cuantificar el volumen del que se podría disponer para el procedimiento reconstructivo.

En nuestro Servicio damos prioridad reconstructiva al tejido autólogo sobre el uso de hueso sintético, y con la técnica que hemos presentado, lo que pretendemos es poner a disposición del cirujano plástico un método reconstructivo más a tener en cuenta en su armamentario quirúrgico. 
